\author{
Military Technical College \\ Kobry El-Kobba, \\ Cairo, Egypt
}

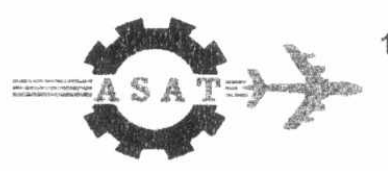

11-th International Conference on Aerospace Sciences \& Aviation Technology

\title{
Performance Improvement of CDMA IRIDIUM Satellite Systems
}

\author{
SHERIEF SAMY EMAM*
}

\begin{abstract}
Applying different coding techniques to the Gaussian mixture traffic model. The effect of convolutional and Turbo coding on the probability of error and the capacity are investigated for CDMA IRIDIUM Low Earth Orbit Satellite (LEOS) systems. The model employed assumes a contaminated Gaussian traffic model. The conventional Gaussian distribution can be considered as a special case. The probability of error decreases using convolutional and turbo coding respectively. Furthermore, the capacity increases. Slight varitions around these values are obtained depending on the traffic nonuniformity. Thus Turbo coding improves the performance of the CDMA LEOS systems to a great extend.
\end{abstract}

\section{KEYWORDS}

Low Earth Orbit Satellite Communication

\footnotetext{
PhD. Egyptian Armed Forces
} 


\section{INTRODUCTION}

Low earth orbit satellite communication systems are one of the most appropriate systems to offer personal communications (PC) [1-3]. They can also provide additional advantages for the global communication networks, e.g., small propagation delay and loss, and high elevation angle in high latitude [4]

One of the most recent candidates for establishing the multiple access in LEOS systems is Code Division Multiple Access (CDMA). CDMA has higher capacity than TDMA and FDMA if voice activity and frequency reuse by spatial isolation are employed [5]. The non-uniform distribution of the traffic is a normal feature of our globe. However there are only few studies on the effect of this non-uniformity of the traffic on the capacity of LEOS systems. Capacity analysis of LEOS communication systems with traffic non-uniformity was considered in [6]. In this later paper, the traffic model was assumed to have a Gaussian probability density function with variance $\omega^{2}$, which represents the traffic non-uniformity. The model represents the case of an isolated city. Due to the relative large area coverage of a LEOS, more than one inhabited area can exist in the coverage area of three consecutive satellites. In this case the Gaussian distribution will not be a good choice to represent the traffic nonuniformity. A (rather) more suitable distribution for a lot of practical cases is the Gaussian mixtures, which is considered in this paper.

This paper suggests a general traffic model that can resemble specific areas in the globe. It also discusses the effects of traffic non-uniformity and coding on the performance of the LEOS communication system employing CDMA scheme. In section II we define the suggested traffic model. Section III reviews expressions for the probability of error for convolutional, and turbo coding techniques. Section IV discusses how the capacity calculation is obtained. Effects on the probability of error and capacity are introduced in Section V. Section VI displays the numerical results. Finally, conclusions are included in Section VII.

\section{THE CONTAMINATED GAUSSIAN TRAFFIC MODEL}

In LEO satellite systems, the satellites are organized on a multiple orbit configuration. in the proposed system for example CDMA IRIDIUM, consists of 66 satellites are organized in 6 orbits, each with 11 satellites. For the sake of simplicity and to make the effect of traffic non-uniformity more clear we will consider the simple 2dimensional model with a single orbit as in [6].

In this model, shown in Fig. 1, the arc represents an area on the earth [7]. The figure shows the coverage and interference areas of each satellite. The coverage area is specified by the minimum elevation angle $\left(\theta_{\min }\right)$. The interference area of a satellite is specified by the final line of sight of that satellite. An area covered by two satellites will be denoted by "double coverage area". $B_{i}$ is the angular position of the $i^{\text {th }}$ satellite measured from the center of the earth. To analyze the effect of traffic non-uniformity, we define a general traffic distribution as the sum of Gaussian distributions with different parameters $[6,7]$

$$
P(\alpha)=A \sum_{i=-M}^{M} \frac{\varepsilon_{i}}{\omega_{i}} \exp \left(\frac{-\left(\alpha-\mu_{i}\right)^{2}}{2 \omega_{i}^{2}}\right), \quad|\alpha| \leq \pi, \quad 0 \leq \varepsilon_{i} \leq 1
$$

Where $\alpha$ is the angular distance of any user from the origin measured by the angle at the center of the earth in radians, $\mu_{i}$ is the center of the $i^{\text {th }}$ populated area, $\omega_{i}$ is the nonuniformity parameter of the $i^{\text {th }}$ populated area and $\varepsilon_{i}$ is the weight of the $i^{\text {th }}$ populated area relative to the total traffic load. The number of populated areas will 
depend on " $M$ " and the values of $\varepsilon_{i}$ as will be shown later. The factor, $A$ is related to the total traffic load (total number of users) in the observed area and is given by $[6,7]$ :

$$
A=B\left[\int_{-3 \pi / N_{s}}^{3 \pi / N} \sum_{i=-M}^{M} \frac{\varepsilon_{i}}{\omega_{i}} \exp \left[\frac{-\left(\alpha-\mu_{i}\right)^{2}}{2 \omega_{i}^{2}}\right] d \alpha\right]^{-1}
$$

Where the constant $B$ is the total traffic load for the three satellites between $3 \pi / N_{s}<\alpha<3 \pi / N_{s}, N_{s}$ is the number of satellites in one orbit. For the sake of simplicity we will assume that $\omega_{i}=\omega$ for all $i$.

Here we examine how the degree of the traffic nonuniformity affects the performance of the system. In the case where one satellite, say, the ith one, is above the traffic peak, the signal to interference ratio (SIR) at the ith satellite becomes[7]

$$
\operatorname{SIR}_{i}=1 / 2\left[\int_{0}^{\pi / N} P(\alpha) d \alpha+\int_{\pi / N_{*}}^{\beta_{l}} P(\alpha) L_{i+1}^{2}(\alpha) L_{i}^{-2}(\alpha) d \alpha\right]
$$

Where the distance $\mathrm{Li}(\alpha)$ (measured in meters) between the $\mathrm{i}^{\text {th }}$ satellite and the earth station at the angular offset $\alpha$ is giveven by $[6,7]$ :

$$
\begin{gathered}
L_{i}(\alpha)=\left[\left(R+h-R \cos \left(\beta_{i}-\alpha\right)\right)^{2}+R^{2} \sin ^{2}\left(\beta_{1}-\alpha\right)\right]^{1 / 2} \\
i=1,2, \ldots, N
\end{gathered}
$$

and the angle $\beta_{i}$ is the geometric interference limit for each of the satellites in $a$ smooth spherical earth is given by $[6,7]$ :

$$
\beta_{l}=\cos ^{-1}\left(\frac{R}{R+h}\right)
$$

Where $h(\mathrm{~km})$, is the altitude of the satellites and $R$ is the average radius of the earth and it is equal to $6378 \mathrm{Km}$.

Next, we shall consider three cases of the distribution of the users.

\section{A. The Conventional Gaussian Distribution}

In this case $P(\alpha)$ is given by:

$$
P(\alpha)=\frac{A}{\omega} \exp \left(-\alpha^{2} / 2 \omega^{2}\right), \quad|\alpha| \leq \pi
$$

Which is the same distribution assumed in $[6,7]$, and it can be obtained from equation (1) by putting $\mathrm{M}=0, \varepsilon_{i}=1, \mu_{0}=0$.

\section{B. The Bimodal Conventional Gaussian Distribution}

In this case, the conventional contaminated Gaussian distribution is given by:

$$
\begin{gathered}
P(\alpha)=\frac{A}{\omega}\left[\varepsilon \exp \left[\frac{-(\alpha-\mu)^{2}}{2 \omega^{2}}\right]+(1-\varepsilon) \exp \left[\frac{-(\alpha+\mu)^{2}}{2 \omega^{2}}\right]\right], \\
|\alpha| \leq \pi, \quad 0 \leq \varepsilon \leq 1
\end{gathered}
$$

when $\mu=0$ and for any value of $\varepsilon$ in the above equation, equation (6) is obtained. 
C. The Trimodal Conventional Gaussian Distribution

In this case $P(\alpha)$ is given by:

$$
\begin{aligned}
& P(\alpha)=\frac{A}{\omega}\left[\varepsilon_{1} \exp \left[\frac{-(\alpha-\mu)^{2}}{2 \omega^{2}}\right]+\varepsilon_{\exp }\left[\frac{-\alpha^{2}}{2 \omega}\right]+\varepsilon^{2} \exp \left|\frac{(\alpha+\mu)^{2}}{2(\omega)}\right|\right] \\
& |\alpha| \leq \pi, \quad 0 \leq \mathcal{E}_{1}, \mathcal{E}_{2}, \mathcal{E}_{i} \leq 1, \quad \varepsilon_{i}+\mathcal{E}_{2}+\varepsilon_{i}=1
\end{aligned}
$$

Equations (7) and (8) are suggested distributions which can represent in the real world the case of two or three populated areas respectively.

\section{PROBABILITY OF ERROR FOR CONVOLUTIONAL AND TURBO CODING ALGORITHMS}

In this section we will review the expressions for the probability of error without coding and when using block, convolutional and turbo coding techniques

\section{A. Without Coding}

The bit error rate for BPSK is given by [8]

$$
P_{e}=Q\left(\sqrt{\frac{2 E_{1}}{(N+I)}}\right)
$$

Where $E_{b} /(N+I)$ is the bit energy-to-noise plus interference ratio.

\section{B. Convolutional Coding}

Convolutional codes (C) are powerful coding schemes for wireless mobile communication systems. We can define the constraint length as the number of shift register in the convolutional encoder.

The probability of bit error when using Viterbi decoding is given by [8]:

$$
P_{e} \leq \frac{1}{k} \sum_{d=d_{\min }}^{\infty} u(d) P_{2}(d)
$$

where $P_{2}(d)$ represents the pair wise error probability, $k$ is the constraint length, $a(d)$ is the number of bit errors in all adversaries at distance $d$ from the correct is the number of selecting the incorrect path for $d$ even and hard decision decoding is given by [8]

$$
P_{2}(d)=\frac{1}{2}\left(\begin{array}{c}
d \\
d / 2
\end{array}\right)[P(1-P)]^{d / 2}+\sum_{k=1+d / 2}^{d}\left(\begin{array}{l}
d \\
k
\end{array}\right) P^{k}(1-P)^{d-k}
$$

where $P$ is the probability of a bit error without coding. and for $d$ is odd is given by:

$$
P_{2}(d)=\sum_{k=(1+d) / 2}^{d}\left(\begin{array}{l}
d \\
k
\end{array}\right) P^{k}(1-P)^{d-k}
$$

\section{Turbo Coding}

A turbo encoder consists of two parallel concatenated convolutional encoders called coonstituent codes (Recursive Systematic encoders (RSC)) separated by an interleaver, with an optional puncturing mechanism [9].

The probability of error for Turbo coding $(T)$ is bounded by: 


$$
P_{e} \approx \max _{w \geq 2}\left[\frac{w n_{w}}{k} Q\left(\sqrt{\frac{2 R d_{w, \min }^{\pi} E_{h}}{(N+I)}}\right)\right]
$$

where $n_{w}$ and $d_{w, \min }^{T C}$ are functions of the particular interleaver employed, $w$ is the weight of the data word, $d_{w, \min }^{T C}$ is the minimum weight turbo codeword produced by $w$. $R$ is the code rate, and $k$ is each block of incoming data bits.

\section{CAPACITY CALCULATION}

An important performance measure in LEOS is the system capacity. To calculate the capacity, we first determine the ratio of the bit energy-to-noise plus interference density for the $i^{\text {th }}$ satellite [6]:

$$
\left(E_{b} /(N+I)\right)_{i}=\frac{B W / R_{b}}{\left.\left(I_{i} / S_{i}\right)+(I) / S_{i}\right)}
$$

where the numerator is the ratio of the total bandwidth $(B W)$, to the information bit rate $\left(R_{b}\right)$, and the denominator is the total interference-to-signal ratio plus the ratio of background noise $(\eta)$, to signal. The total interference reaching the $i^{\text {th }}$ satellite $l_{i}$ is proportional to the total traffic load $B$. Therefore the maximum amount of traffic that the system can support for a given condition can be denoted as:

$$
B_{i}=\frac{I_{i}}{\left[I_{i}\right]_{B=1}}
$$

where $\left[l_{i}\right]_{B=1}$ means the interference $l_{i}$ calculated at $B=1$. Solving (14) for $l_{i}$ and substituting it in (15) we have

$$
B_{i}=\left[\frac{B W / R_{b}}{\left(E_{b} /(N+I)\right)}-\frac{\eta}{S_{i}}\right] \frac{S_{i}}{\left[I_{i}\right]_{B=1}}
$$

From this equation, we can derive the maximum traffic $B_{i}$ (capacity) for a certain $E_{b} /(N+l)$ on the $i^{\text {th }}$ satellite for a given $B W, R_{b}, \eta$, and $S_{i}$. The capacity is dependent on $E_{b} /(N+l)$.

\section{EVALUATION OF PROBABILITY OF ERROR AND THE CAPACITY FOR LEOS WHEN CODING IS EMPLOYED}

In the following, we are going to show how the probability of error and the capacity are evaluated using convolutional and turbo coding techniques.

\section{A. Convolutional Coding}

Taking the probability of error without coding for a certain ${ }^{\prime}$ ) and substituting in equation (11) or equation (12) and equation (10). Then solving equation (10) using numerical solution we get the corresponding $E_{b} /(N+1)$. Finally by substituting this value in equation (16) we get the required capacity.

\section{B. Turbo Coding}

Taking the probability of error without coding for a certain $\omega$ and substituting in equation (13). Then solving equation (13) using numerical solution we get the corresponding $E_{b} /(N+l)$. Finally by substituting this value in equation (16) we get the required capacity 


\section{NUMERICAL RESULTS}

The probability of error and the capacity are calculated for the three consecutive satellites $S_{1}, S_{2}$ and $S_{3}$ for the case of one, two and three populated areas respectively assuming that all received satellites power are equal in each case we study the effect of coding.

For a certain $\omega$ we calculate SIR from equation (3), from equation (14), we obtain $E_{b} /(N+1)$ and the probability of error from equation (9) without coding.

A convolutional code of constraint length 7, minimum distance 10 and rate $1 / 2$ is considered, and hard decision Viterbi decoding is assumed. For turbo code case the performance of a rate $1 / 2$, for two different interleavers of size $k=1000, w=3$, and $d_{11 \text { min }}^{\prime \prime}=9$ is considered.

The probability of error for each satellite for the three distributions of users under consideration is shown in figures (2), (3) and (4). It is clear from these figures that the turbo code outperforms convolutional coding techniques for the three satellites. A probability of error of $10^{-7}$ or less can be obtained depending on the uniformity of the traffic.

From the above three figures, in the uniform case $(\omega>5)$ an improvement from 0.027 to $3 \times 10^{-5}$ and $2 \times 10^{-11}$ has been obtained for convolutional code and turbo code respectively. Furthermore, for large $\omega$. Pe is the same for the three consecutive satellites for all cases under consideration.

The effect of coding on the capacity for each satellite in the case of one, two and three populated area is investigated in figures (5), (6) and (7). In these Figures, $B W=$ $1.25 \mathrm{MHz}, R_{b}=8 \mathrm{~Kb} / \mathrm{s}$ and $S / \eta=-1 \mathrm{~dB}$. Using the turbo code, the capacity of the satellite increases 4 to 5 times or more depending on the uniformity of the traffic. In the uniform case, the capacity increases from 14 when no coding is used to 83 and 100 when using convolutional, and turbo coding respectively.

From these figures, it is clear that for turbo coding case, the capacity is identical for the three adajacent satellites and is independent neither on $\omega$ nor the users distribution. For convolutional coding case, the capacity is identical for the three adajacent satellites at large $\omega$, while it is dependent on the users distributions Furthermore, varying $\varepsilon, \mu$ and $\omega$ controls mainly the number of users in each service area, thus affects the performance of each satellite. The three adajacent satellites will be balanced, that is have the same performance, if they can offer service to the same number of users. Otherwise, there will be an unbalance between the three satellites resulting in having sparse traffic satellite (STS) and dense traffic satellite (DTS) corresponding to better and worse performance respectively.

\section{CONCLUSIONS}

A Gaussian mixture traffic model was introduced, it was shown that this distribution can fit specific cases of the globe, by the proper choice of the parameters $\varepsilon_{i}$ and $\mu$. Previous model is considered as a special case of this suggested model. In the uniform case we conclude that the probability of error decreases from 0.027 to $3 \times 10^{-5}$ and $2 \times 10^{-11}$ using convolutional and turbo coding respectively. Furthermore, the capacity increases from 14 to 83 and 100 . Slight varitions around these values are obtained depending on the traffic nonuniformity. Thus Turbo coding improves the performance of the LEOS system to a great extend. At large $\omega$, abalanced situation is obtained. That is, the same probability of error and identical capacity are 
obtained for the three satellites under any particular employed coding techniques. Furthermore, these values are independent on the users distribution.

\section{REFERENCES}

[1] D. Chakraborty, "Survivable communication concept via multiple low earthorbiting satellites, "IEEE Trans. On AES. Vol. 25, no. 6, pp. 879-889,1989.

[2] J.Kaniyil, J.Takei,S. Shimamoto, Y. Onozato, T. Usui, I. Oka, T. Kawabata, "A global message network employing low earth-orbiting satellites, "IEEE Jour. On SAC, vol. 10, no. 2, pp. 418-428, 1992.

[3] R. J. Leopold, "The Iridium communication system, "in Proc. Of the ICCS/ISITA. Singapore, pp. 451-455, 1992

[4] M. Katayama, A. Ogawa, and N. Morinage," Satellite communication systems with low earth orbits and the effect of Doppler shift," IEICE Trans., vol. J67- B-II, no. 5, 1993, PP. 382-390.

[5] K. S. Gilhousen, I. M. Jacobs, R. Padovani. A. J. Viterbi, L. A. Weaver, Jr andC E. Wheatly III, "On the capacity of a cellular CDMA system, "IEEE Trans. Veh. Tech., vol. 40, no. 2, pp. 303-312, 1991

[6] A. Jamalipour, M. Katayama, T. Yamazato, and A. Ogawa,"a performance analysis on the effects traffic nonuniformity in low earth-orbit satellite communication systems," in Proc. 16th Symp. Inform. Theory. Applicat. (SITA'93) (Japan), Vol. 1, 1993, PP. 203-206.

[7] A. Jamalipour et al., "performance of an integrated voice/data system in nonuniform traffic low earth orbit satellite communication systems," IEEEJ. Select. Areas Commun., Vol. 13, No. 2, 1995, PP. 465-473.

[8] Pritchard, W. L., H. G. Suyderhoud, and R. A. Nelson, Satellite Communication Engineering, 2nd ed, Englewood Cliffs, NJ: Prentice- Hall, 1987.

[9] Werner, M., et al., "Analysis of System Parameters for LEO/ICO-Satellite Communication Networks," IEEE J. Select. Areas Commun., Vol. 13, No. 2. 1995, pp.371-381.

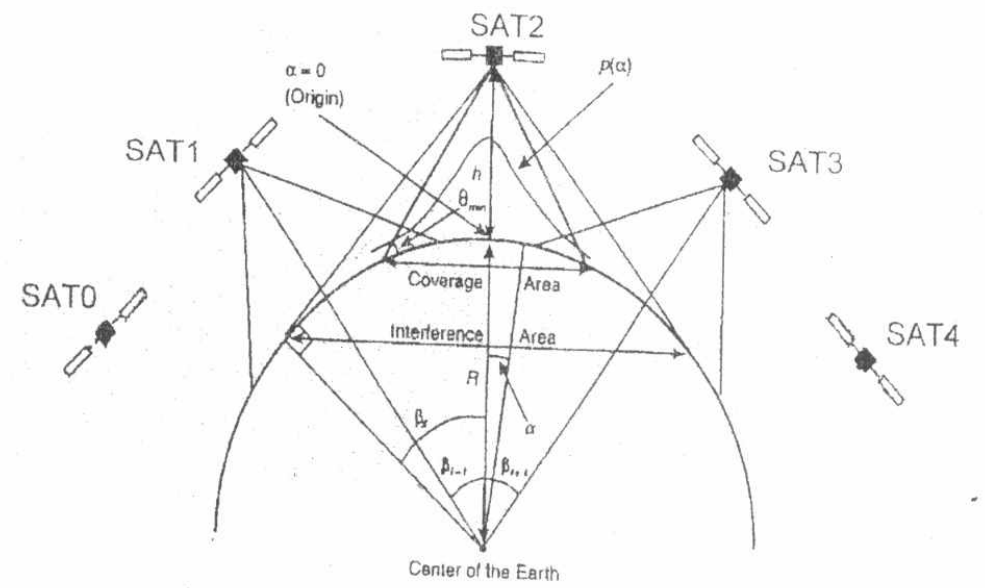

Fig. 1. The System model 


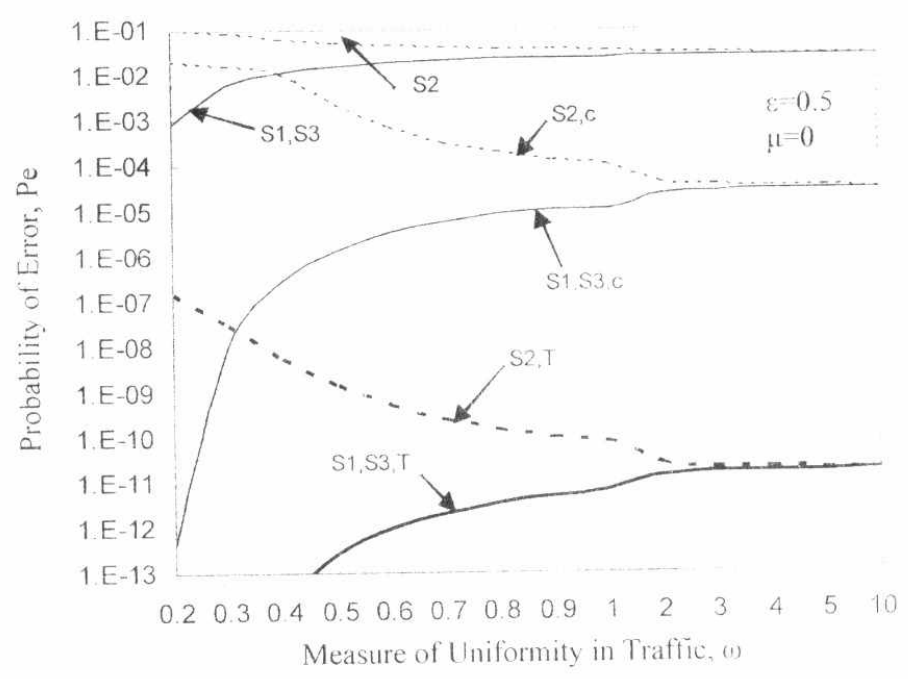

Fig.2. Probability of error in the case of one populated area without coding, with convolutional code $(\mathrm{C})$ and with Turbo code $(\mathrm{T})$ for the three adjacent satellites $\left(\mathrm{S}_{1}, \mathrm{~S}_{2}, \mathrm{~S}_{3}\right)$

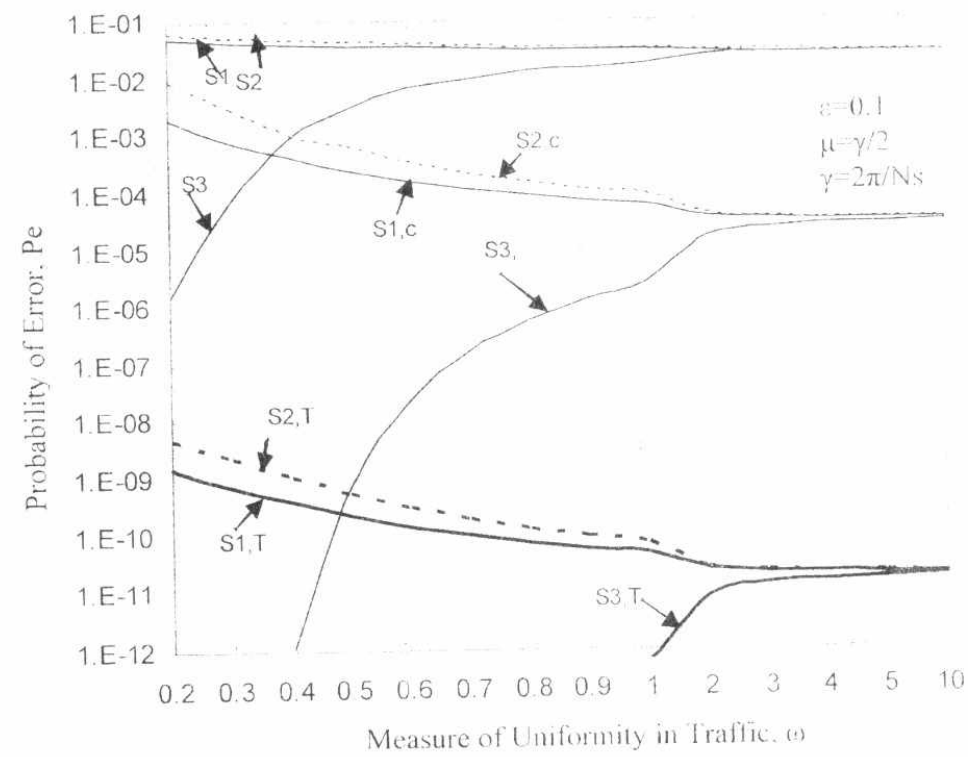

Fig. 3. Effect of coding on the probability of error in the case of two populated areas 


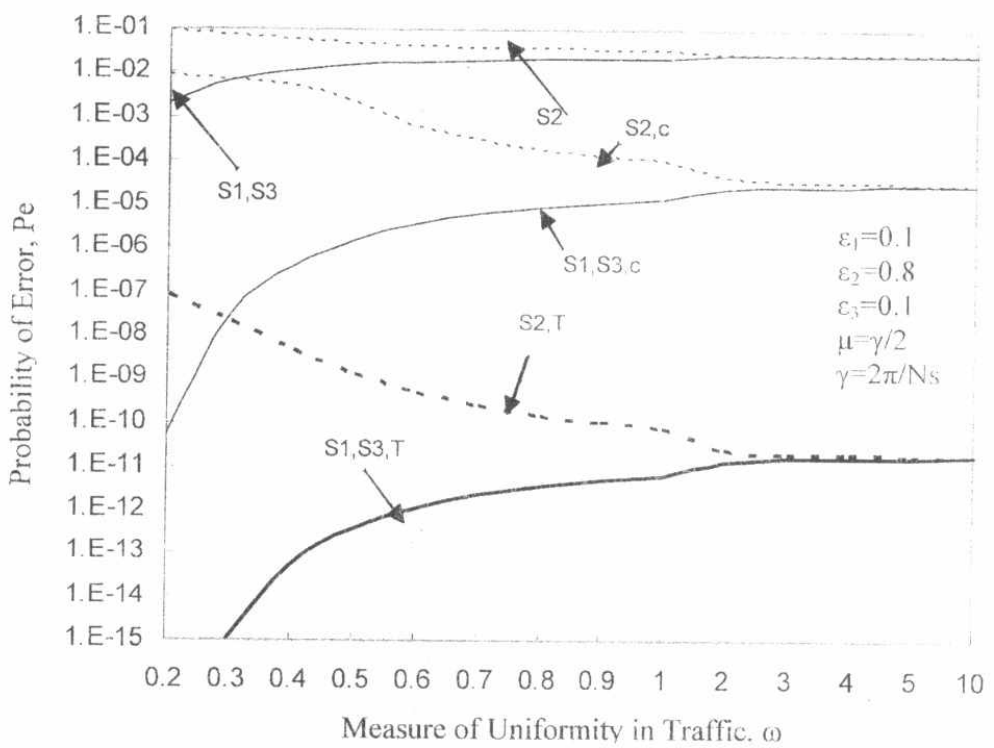

Fig.4. Effect of coding on the probability of error in the case of three populated areas

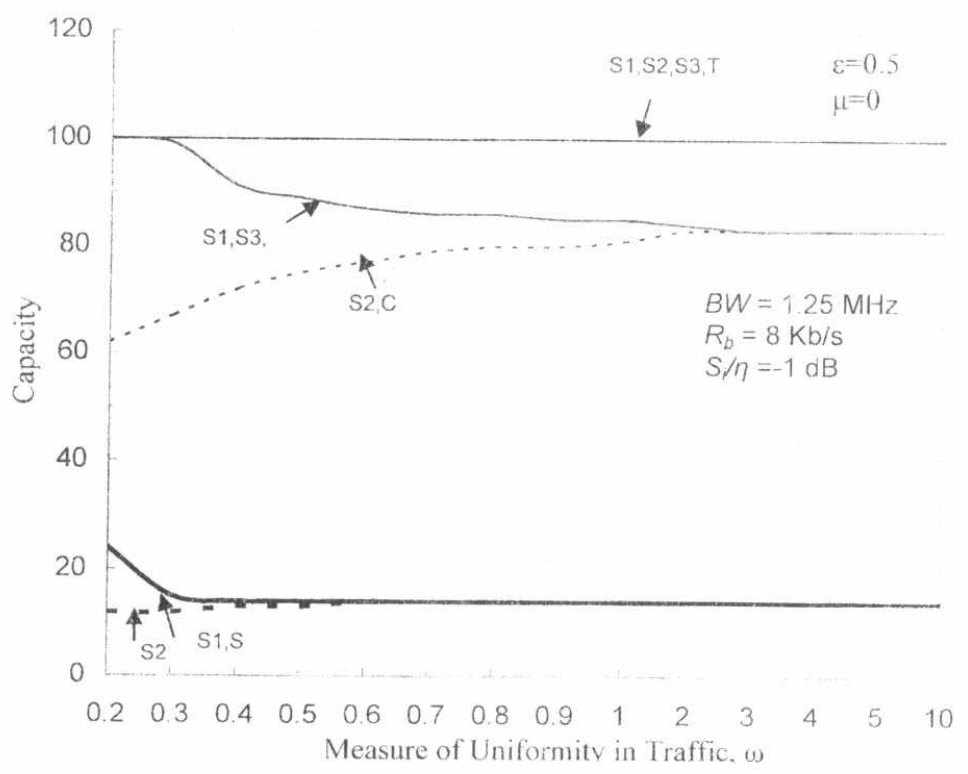

Fig.5. Effect of coding on the capacity in the case of one populated area 


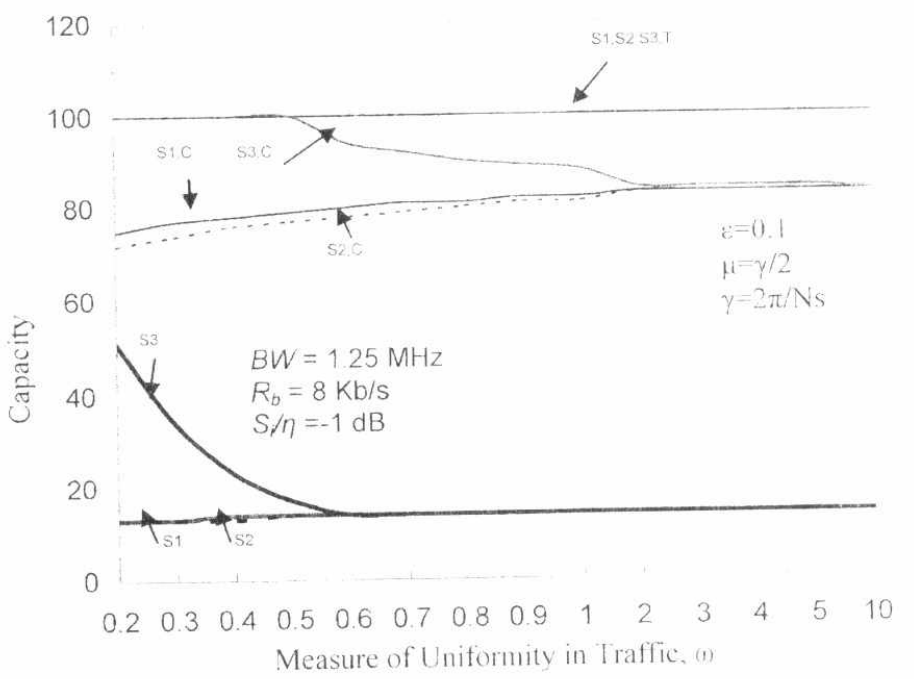

Fig.6. Effect of coding on the capacity in the case of two populated areas

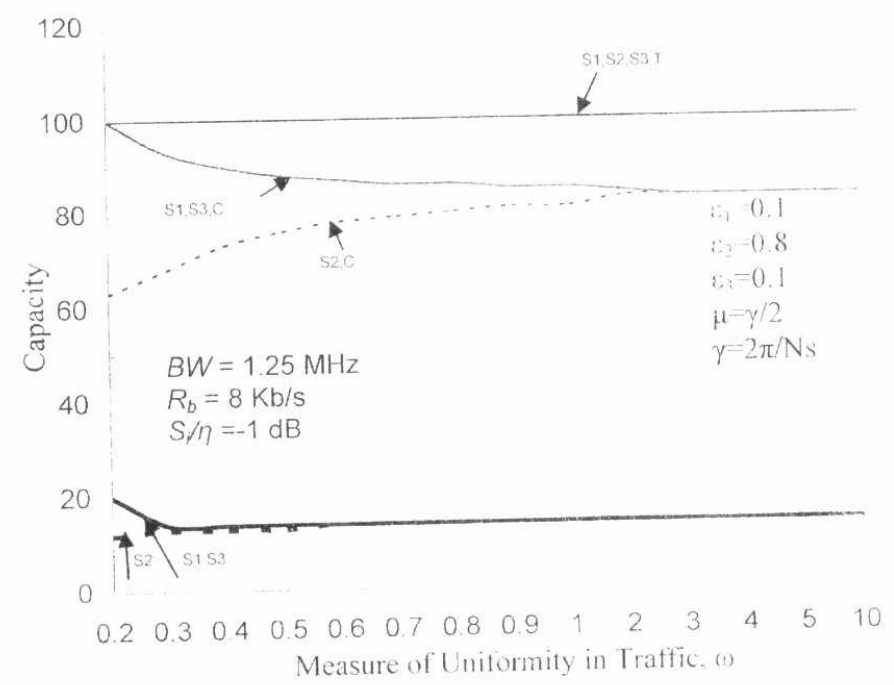

Fig. 7. Effect of coding on the capacity in the case of three populated areas 